\title{
Tecnología de la Información y Comunicación (TIC) como estrategias de marketing y su contribución a la competitividad del sector turístico hotelero de la Ciudad de Estelí, Nicaragua, en el I semestre del 2016
}

\author{
Samaria Ilú Alonso Valenzuela ${ }^{1}$ \\ Flor Idalia Lanuza ${ }^{2}$ \\ Natalia Golovina ${ }^{3}$
}

\section{RESUMEN}

La presente investigación se realizó en la Ciudad de Estelí, año 2016. Participaron turistas y gerentes y/o propietarios de los hoteles inscritos en el Instituto Nicaragüense de Turismo, delegación de Estelí. El objetivo de la investigación ha sido evaluar el uso y aplicación de las Tecnologías de la Información y Comunicación (TICs) como estrategias de Marketing y su contribución a la competitividad del sector turístico hotelero de la cuidad. Para ello, se realizó una caracterización del sector turístico hotelero; así mismo se identificó y valoró la aplicación de las TICs en el sector, lo cual permitió analizar la contribución a la competitividad. Con los resultados obtenidos se realizó un FODA, que sirvió de insumo para elaborar la propuesta de estrategias. El estudio se justificó por su conveniencia dado que cuenta con valor teórico, utilidad práctica, y relevancia social. Metodológicamente aborda la perspectiva desde un enfoque cuantitativo, analiza y vincula datos con su aportación teórica, de tipo aplicada, se basa en un paradigma pragmático, de corte transversal y muestreo no probabilístico; el universo constó de 50 turistas y 19 propietarios y/o gerentes. La información se recolectó mediante encuestas a los turistas y a los propietarios y/o gerentes, se procesaron haciendo uso del programa SPSS versión 20.0. Los resultados indican que la mayoría de los hoteles hacen uso y aplican alguna estrategia de marketing digital, la cual aporta a su competitividad. Se presentan debilidades como fortalezas que se pueden mejorar y optimizar para aprovechar y contrarrestar las oportunidades y amenazas provenientes del entorno.

Palabras claves: Tecnologías de la Información y Comunicación, Estrategias de Marketing, Competitividad y Sector Turístico Hotelero.

Recibido: 20 de septiembre de 2016

Aceptado: 24 de octubre de 2016

1 Egresada de la maestría en Gestión Sostenibilidad y Calidad de las MIPYMES, docente en UNAN-Managua/ FAREM-Estelí. Correo electrónico: sama_alvalen@yahoo.es

2 Tutora de investigación, Máster en Economía. Docente en UNAN-Managua/FAREM-Estelí. Correo electrónico: florlan2@yahoo.com

3 Asesora metodológica. Doctora en Ciencias Sociales de la Universidad del Zulia. Docente en UNAN-Managua/ FAREM-Matagalpa. Correo electrónico: natygolovina@gmail.com 


\title{
Information and Communication Technologies (ICT) as marketing strategies, and their contribution to the competitiveness of the hotel- tourist sector in Estelí, Nicaragua, during the first semester of 2016
}

\begin{abstract}
The present research was carried out in Estelí, during 2016, where tourists and managers and/ or owners of the hotels registered to the National Institute of Tourism of Estelí participated. The research aimed to evaluate the use and application of ICT tools as marketing strategies, and their contribution to the competitiveness of the hotel-tourism sector in Estelí. To that end, a characterization of the hotel-tourism sector was made. Similarly, the application of the use of ICT resources was identified and valued. This allowed the researchers to analyze its contribution and competitiveness. A SWOT was made from the results obtained, and a proposal of strategies was designed. The study was justified due to its convenience, as it has a theoretical value, practical usage and social relevance. From a methodological perspective, it followed a qualitative approach; the research was applied and belonged to the interpretative paradigm. The research universe was made up by 50 tourists and 19 owners/managers. Data were collected through surveys to tourists and owners/managers, and processed using the software SPSS, version 20.0. The main research results indicate that the majority of the hotels use some strategy of digital marketing, which improves their competitiveness. However, they have some weaknesses that can be improved and optimized to take advantage of and prevent threats from the environment.
\end{abstract}

Key words: ICT Tools, marketing strategies, competitiveness, hotel-tourism sector. 


\section{INTRODUCCIÓN}

La presente investigación se enmarca en el estudio del uso y aplicación de las tecnologías de la información y comunicación como estrategias de Marketing y su contribución a su competitividad en el sector turístico hotelero de la ciudad de Estelí.

Para la elaboración de esta investigación se consultaron dos fuentes de información, los turistas que visitan la ciudad y los gerentes y/o propietarios de los hoteles delimitándolo a solo aquellos que están inscritos en el Instituto Nicaragüense de Turismo (INTUR).

Para dar respuesta a los objetivos planteados, se estructuro los ejes teóricos, tales como: sector turístico hotelero, TICs, Marketing digital y estrategias de Marketing digital, mismas que están plasmados en el cuadro de operacionalización de variables. Cada una de estas variables se analizó de manera que permitiera dar salida a cada objetivo planteado.

Con la información obtenida se realizó una caracterización del sector turístico de la ciudad de Estelí en la cual se analizó la demanda y la oferta turística; además se identificó el uso y aplicación de las TICs como estrategias de Marketing y su aportación a la competitividad del sector turístico hotelero. También se analizó las principales debilidades, fortalezas, amenazas y oportunidades que posee el sector, lo que permitió la elaboración de propuestas de estrategias que los hoteles pueden aplicar para la mejora de su competitividad, así mismo, se detallan las conclusiones y recomendaciones de la investigación.

\section{MATERIALES Y MÉTODOS}

De acuerdo al problema, propósito y objetivos de investigación, es un estudio cuantitativo. Se basa en un paradigma pragmático, ya que las relaciones entre el investigador y el fenómeno o participantes del estudio están determinadas por lo que el investigador considera como apropiado para cada estudio en particular y el método depende del planteamiento específico del estudio.

Según su aplicabilidad es una investigación aplicada, porque analiza la problemática del sector hotelero de la ciudad de Estelí en relación al uso y aplicación de las tecnologías de la información y comunicación como estrategia de marketing digital.

Según el tiempo, es una investigación transaccional o de corte transversal, ya que se recogerán los datos en tiempo único en un momento determinado, así mismo se analizará la contribución de las TIC en la estrategia competitiva como herramienta de marketing digital en el sector hotelero.

Para este estudio dado la naturaleza de la información, se necesitó dos universos o población de estudio, los cuales son:

- Demanda turística: 50 turistas como promedio mensual inscritos en la Delegación del Instituto Nicaragüense de Turismo. (Ramírez, 2016 ).

- Oferta turística hoteles: 19 hoteles que están inscritos en el Instituto Nicaragüense de Turismo: Alameda, Camilo`s, Campestre Estelí, Cualitlan, Don Vito, El Chico, El Mesón, Estelimar, Hex Estelí, La Campiña, Las Américas, Las Cornisas, Los Arco, Mery, Panorama \# 1, Panorama \# 2, Puro Estelí , Los Altos y Casa Vínculos

Para definir la muestra se definieron dos criterios:

- Turistas que visitan la ciudad de Estelí.

- Informantes claves de la empresa (Propietarios de los hoteles o gerentes).

Para definir el tamaño de la muestra se aplicó un censo de cantidad de turistas inscritos como promedio mensual en la delegación de INTUR, y un censo a los 19 hoteles inscritos en la INTUR. 
El tipo de muestreo fue no probabilístico, ya que los elementos no dependen de la probabilidad, sino de causas relacionadas con las características de la investigación o de quien hace la muestra.

Para llevar a cabo esta investigación también se utilizó el método deductivo que es un método científico que considera que la conclusión se haya implícita dentro las premisas. Esto quiere decir, que las conclusiones son una consecuencia necesaria de las premisas: cuando las premisas resultan verdaderas y el razonamiento deductivo tiene validez, no hay forma de que la conclusión no sea verdadera. Se desciende de lo general a lo particular, de forma que partiendo de enunciados de carácter universal y utilizando instrumentos científicos, se infieren enunciados particulares. (Hernández Sampierí, Fernández Collado, \& Baptista, 2010).

El conocimiento deductivo permite que las verdades particulares contenidas en las verdades universales se vuelvan explícitas, esto es, a que partir de situaciones generales se lleguen a identificar explicaciones particulares contenidas explícitamente en las situaciones generales. Así, de la teoría general acerca de un fenómeno o situación, se explican los hechos o situaciones particulares. (Mendez A., 2009).

Para la recolección, procesamiento y análisis de los datos obtenidos se utilizó el método empírico. El método empírico consiste en la recolección de datos, en los cuales a base de teoría se derive una conclusión científica, empírico significa algo que ha surgido de la experiencia directa de las cosas, su fundamento radica en la percepción directa del objeto de investigación y del problema (Tamayo, 1994).

Para la recolección de datos de esta investigación se utilizó la encuesta y la entrevista semi-estructurada. Se diseñaron dos instrumentos de encuesta, una dirigida a los turistas que visitan la ciudad de Estelí con el propósito de realizar su caracterización; y otra para los propietarios o gerentes de los hoteles, lo cual permitió conocer el uso y la aplicación de las tecnologías de la información y la comunicación como estrategia de marketing digital y su contribución a la competitividad.

El proceso de validación de los instrumentos fue realizado por dos maestros que revisaron y dieron sugerencias sobre los instrumentos aplicados en el estudio. Esta validación fue de mucha importancia ya que permitió realizar las correcciones a los instrumentos utilizados en el estudio.

Antes de iniciar el desarrollo investigativo de este trabajo fue necesario conocer la principal problemática del sector turístico, así mismo, de la existencia de estudio que se relaciona con la temática elegida, con el fin de tener certeza de que la investigación sea de carácter innovador.

Luego de definir el tema se requirió consultar algunas fuentes de información como: libros, sitios web, tesis, entre otros; lo que facilitó el desarrollo teórico conceptual importante y coherente para diseñar el marco teórico de la indagación, utilizando como referencia en todo el proceso.

Para el proceso de recolección de los datos se acudió a la delegación de INTUR en la ciudad de Estelí para obtener información sobre el número de hoteles y el número de turistas que visitan la ciudad.

Cabe mencionar que no existe una estadística del número de visitantes en la ciudad, INTUR solo posee un registro de los visitantes que visitan la delegación lo cual llevan un registro mensual de esas visitas.

Posteriormente, se procedió a aplicar la entrevista a delegada de INTUR, las encuestas a los turistas de la ciudad y a propietarios o gerentes de los hoteles.

En esta etapa se procesó la información obtenida con el programa SPSS versión 22.5 versión libre de internet, con la implementación de los instrumentos a cada 
empresario, logrando diseñar las tablas de contingencia y tabular los gráficos para la presentación del análisis de resultados, conclusiones y recomendaciones finales.

\section{RESULTADOS Y DISCUCIÓN}

El sector hotelero está conformado según datos del INTUR por 19 hoteles, los cuales cumplen con las normas técnicas del INTUR para su categorización.

En la investigación realizada sobre tecnología de la información y comunicación como estrategias de marketing y su contribución a la competitividad del sector turístico hotelero de la Ciudad de Estelí, para ello, se emplearon diversos métodos y herramientas incluidas la encuesta y entrevista, teniéndose los siguientes resultados:

\section{Caracterización del sector turístico hotelero de la ciudad de Estelí.}

La importancia de realizar una caracterización del sector turístico es poder identificar el mercado en el que actúa el sector turismo hotelero en Estelí, para así poder proponer estrategias que se adecuen a las características de los demandantes y oferentes turísticos del sector hotelero.

A partir de los datos obtenidos en la encuesta dirigida a turistas y al sector hotelero de la ciudad de Estelí se realizó una caracterización del sector turístico hotelero, analizando la demanda y oferta turística de la ciudad de Estelí.

La demanda turística está relacionada con el consumo por el visitante antes, durante y después de la realización de un viaje.

Los mercados consisten en compradores, los cuales difieren en sus deseos, recursos, ubicación, actitudes o prácticas de compra, por medio de la segmentación de mercado, las empresas pueden dividir mercados grandes en mercados más pequeños, a los cuales se puede llegar de manera más eficaz con productos congruentes con sus necesidades únicas (Kotler, 2008).

Para determinar la demanda turística hotelera se analizó los factores socio-demográficos y conductuales de los turistas.

\begin{tabular}{ll}
\hline \multicolumn{2}{c}{ Rasgos sociodemográficos } \\
\hline Género & $54 \%$ mujeres $46 \%$ \\
& Masculino \\
Edad & Menor de 25 años, $28 \%$ \\
& De 26 a 35 años, $42 \%$ \\
& Mayor de 61 años, 10\% \\
Nivel de formación & Estudios secundarios: 40\% \\
& Estudios superiores: $60 \%$ \\
Situación Laboral & Trabajador por cuenta propia, 26\% \\
& Empleado, 50\% Jubilado, 6\% \\
\hline
\end{tabular}

La importancia de identificar si los turistas son hombres y mujeres, radica en que se pueden diseñar productos turísticos y campañas publicitarias o sitios web dirigidos a las mujeres, ya que un poco más del $50 \%$ de demandantes del sector son mujeres, sin dejar de obviar las demandas de los hombres en el mercado hotelero.

Las necesidades y los deseos de los consumidores cambian de acuerdo a la edad. Según los datos obtenidos el $42 \%$ de los turistas se encuentra entre el rango de los 26 a los 35 años, el $30 \%$ es mayor que 35 años y solamente el $28 \%$ es menor que 25 años.

Por otra parte, se puede deducir que las estrategias de Marketing digital y los productos turísticos, tienen que ir dirigidas a un sector relativamente joven, para consolidar este grupo y para fortalecer e identificar otras ofertas que atraigan a turistas de otro rango como son las personas mayores de 61 años enfocados al turista jubilado.

El $60 \%$ de los turistas poseen un nivel de formación de educación superior, afirmando así que el turista 
que visita Estelí es un turista que cuenta con un nivel de formación y con acceso a todos los medios $\mathrm{y}$ tecnologías de información y comunicación.

Una variable socio-demográfica importante a la hora de segmentar el mercado turístico es la situación laboral, ya que la afluencia de los turistas es estacional, y va a ser más alta en la época de vacaciones laborales, ya que como podemos ver el mayor porcentaje de turistas que visitan Estelí son trabajadores por cuenta ajena, es decir que trabajan para alguna empresa o institución, como podemos ver en el siguiente gráfico. En este sentido es importante que el Marketing digital sea más agresivo para las épocas de feriados y vacaciones.

La segmentación conductual divide a los demandantes en grupos con base en sus conocimientos, actitudes, uso y respuesta a un producto o servicio. Muchos mercadólogos piensan que las variables de la conducta son el mejor punto de partida para formar segmentos de mercado (Philip \& Armstrong, 2008).

Mediante el estudio de la demanda turística de Estelí se pudo identificar variables como: la motivación, el tiempo de estadía, los medios que utiliza para la organización del viaje, el tipo de alojamiento, y que aspectos toma en cuenta a la hora de escoger el alojamiento.

\begin{tabular}{|c|c|}
\hline \multicolumn{2}{|c|}{ Rasgos Conductuales } \\
\hline Motivos de la visita & $\begin{array}{l}\text { Vacaciones, } 52 \% \\
\text { Conocer el entorno, 20\% } \\
\text { Otros (visitar familiares, } \\
\text { descanso y trabajo), } 28 \%\end{array}$ \\
\hline Tiempo de estadía & $\begin{array}{l}\text { Entre dos y cinco días, } 38 \% \\
\text { Más de } 10 \text { días, } 32 \%\end{array}$ \\
\hline $\begin{array}{l}\text { Medios de información } \\
\text { sobre la cuidad }\end{array}$ & $\begin{array}{l}\text { Internet, } 52 \% \\
\text { Amistades, } 46 \% \\
\text { Otros (publicidad, agencias de } \\
\text { viaje), } 30 \%\end{array}$ \\
\hline
\end{tabular}

Alojamiento turístico

Hotel, $40 \%$

Hostal, 18\%

Otros (Casa Huésped, Hostal

familiar, Albergue, entre otros), $42 \%$

Búsqueda de alojamiento turístico

Internet, $50 \%$

Recomendaciones de amigos, $34 \%$

Criterios de selección

de alojamiento

Precio, muy importante (62\%)

Accesibilidad, Muy importante $(56 \%)$

Grado de limpieza, muy importante $(58 \%)$

Formas de organización de viaje

Organización propia, $58 \%$

Agencias de viaje, 24\%

Tour operadoras, $18 \%$

Presupuesto

Entre 1 a 249 dólares, 40\%

aproximado de viaje

Los motivos de visitar la ciudad de Estelí, el 52\% son vacaciones, el $20 \%$ su intención es conocer el entorno, y solo un $12 \%$ dijo que su motivación es trabajo y visitar familiares, por lo que tomando en cuenta estos aspectos las estrategias de Marketing digital debe ir enfocada a la promoción de oferta turística complementaria además del servicio de alojamiento, que le ofrezca oportunidades de esparcimiento.

El tiempo de estadía de los turistas en la ciudad de Estelí donde un 38\% va entre dos y cinco día, el $28 \%$ entre 6 y diez días, y un $32 \%$ más de diez días. Así mismo, un $52 \%$ de los turistas indicaron que el medio que utilizan para tener información sobre la ciudad es vía internet, esto es un elemento importante porque demuestra que los propietarios de los hoteles están haciendo uso de las tecnologías de la información y comunicación para promover sus servicios.

Un elemento importante para el sector hotelero, es identificar qué tipo de alojamiento turístico prefieren los turistas, el $40 \%$ se hospeda en hotel y el restante $60 \%$ escogen otro tipo de alojamiento, tales como hostales, casa huéspedes, hostal familiar, albergue, etc. El restante $60 \%$ que se hospeda en otro tipo de 
alojamiento que el sector hotelero no está captando por lo que tendrá que crear estrategias que incentiven la demanda del sector, como por ejemplo estrategias basadas en el precio.

Al momento de escoger el alojamiento, el turista toma en cuenta algunos aspectos, tales como la ubicación, el precio, ubicación, grado de limpieza, grado de confort, sello de calidad e infraestructura. Se puede observar en el gráfico 7, que en mayor porcentaje (62\%), los turistas escogen su alojamiento basándose en el precio, seguido por el grado de limpieza (58\%), la ubicación (58\%), la accesibilidad (56\%) son aspectos que los califican como muy importantes, con menor grado de importancia la infraestructura $(42 \%$ dice que es importante), el 38\% dice que es importante el grado de confort. También es importante mencionar que el
$32 \%$ expresa que no es tan importante tener un sello de calidad.

Los aspectos anteriores son muy importantes a la hora de diseñar el plan de marketing, ya que la estrategia tiene que ir enfocada más que todo en ofrecer buenos precios y descuentos; los cuales se tienen que ver reflejados en la publicidad en línea, ya que en mayor porcentaje $(50 \%)$ los turistas utilizan buscador genérico en internet a través de google, seguido de las recomendaciones de amigos con un 34\%.

La oferta turística hotelera está determinada por la cantidad de servicios que el sector puede ofrecer, la variedad del servicio y la calidad del mismo. En la presente investigación se encontró que el sector está compuesto de la siguiente manera:

\begin{tabular}{|c|c|c|c|c|c|c|c|}
\hline $\begin{array}{l}\text { Año de } \\
\text { fundación }\end{array}$ & Hotel & Categoría & Habitaciones & Camas & Administrativos & $\begin{array}{l}\text { Tipo de } \\
\text { Empresa }\end{array}$ & Página Web \\
\hline 1982 & El Mesón & $\begin{array}{l}1 \text { Una } \\
\text { Estrella }\end{array}$ & 10 & 24 & 2 & $\begin{array}{l}\text { Pequeña } \\
\text { Empresa }\end{array}$ & $\begin{array}{l}\text { www.hotelmeson.esteli. } \\
\text { com }\end{array}$ \\
\hline 1993 & El Chico & $\begin{array}{l}1 \text { Una } \\
\text { Estrella }\end{array}$ & 25 & 41 & 1 & $\begin{array}{l}\text { Pequeña } \\
\text { Empresa }\end{array}$ & No Tiene \\
\hline 1995 & $\begin{array}{l}\text { Panorama } \\
\text { No. } 1\end{array}$ & $\begin{array}{l}2 \text { Dos } \\
\text { Estrellas }\end{array}$ & 48 & 101 & 1 & $\begin{array}{l}\text { Pequeña } \\
\text { Empresa }\end{array}$ & No Tiene \\
\hline 1998 & $\begin{array}{l}\text { Panorama } \\
\text { No. } 2\end{array}$ & $\begin{array}{l}1 \text { Una } \\
\text { Estrella }\end{array}$ & 15 & 28 & 1 & $\begin{array}{l}\text { Micro } \\
\text { Empresa }\end{array}$ & No Tiene \\
\hline 1998 & $\begin{array}{l}\text { Campestre } \\
\text { Estelí }\end{array}$ & $\begin{array}{l}0 \text { Categoría } \\
\text { D }\end{array}$ & 17 & 30 & 1 & $\begin{array}{l}\text { Micro } \\
\text { Empresa }\end{array}$ & No Tiene \\
\hline 2001 & Alameda & $\begin{array}{l}2 \text { Dos } \\
\text { Estrellas }\end{array}$ & 20 & 39 & 1 & $\begin{array}{l}\text { Pequeña } \\
\text { Empresa }\end{array}$ & $\begin{array}{l}\text { www. } \\
\text { alamedanicaragua.com }\end{array}$ \\
\hline 2001 & Estelimar & $\begin{array}{l}1 \text { Una } \\
\text { Estrella }\end{array}$ & 28 & 93 & 3 & $\begin{array}{l}\text { Pequeña } \\
\text { Empresa }\end{array}$ & $\begin{array}{l}\text { www.estelimarasdenic. } \\
\text { org }\end{array}$ \\
\hline 2001 & Cualitlan & $\begin{array}{l}2 \text { Dos } \\
\text { Estrellas }\end{array}$ & 13 & 26 & 1 & $\begin{array}{l}\text { Micro } \\
\text { Empresa }\end{array}$ & No Tiene \\
\hline 2003 & La Campiña & $\begin{array}{l}2 \text { Dos } \\
\text { Estrellas }\end{array}$ & 10 & 12 & 1 & $\begin{array}{l}\text { Micro } \\
\text { Empresa }\end{array}$ & $\begin{array}{l}\text { www.campinaesteli. } \\
\text { com }\end{array}$ \\
\hline 2004 & Los Arcos & $\begin{array}{l}3 \text { Tres } \\
\text { Estrellas }\end{array}$ & 32 & 47 & 3 & $\begin{array}{l}\text { Pequeña } \\
\text { Empresa }\end{array}$ & $\begin{array}{l}\text { www. } \\
\text { hotelosarcosesteli,com }\end{array}$ \\
\hline 2006 & Don Vito & $\begin{array}{l}3 \text { Tres } \\
\text { Estrellas }\end{array}$ & 36 & 43 & 3 & $\begin{array}{l}\text { Pequeña } \\
\text { Empresa }\end{array}$ & $\begin{array}{l}\text { www.hoteldonvitoesteli. } \\
\text { com }\end{array}$ \\
\hline 2007 & Puro Estelí & $\begin{array}{l}1 \text { Una } \\
\text { Estrella }\end{array}$ & 9 & 16 & 2 & $\begin{array}{l}\text { Micro } \\
\text { Empresa }\end{array}$ & $\begin{array}{l}\text { www.hotelpuroesteli. } \\
\text { com }\end{array}$ \\
\hline
\end{tabular}




\begin{tabular}{|c|c|c|c|c|c|c|c|}
\hline 2009 & $\begin{array}{l}\text { Las } \\
\text { Cornisas }\end{array}$ & $\begin{array}{l}1 \text { Una } \\
\text { Estrella }\end{array}$ & 10 & 14 & 1 & $\begin{array}{l}\text { Micro } \\
\text { Empresa }\end{array}$ & No Tiene \\
\hline 2012 & $\begin{array}{l}\text { Las } \\
\text { américas }\end{array}$ & $\begin{array}{l}1 \text { Una } \\
\text { Estrella }\end{array}$ & 21 & 34 & 2 & $\begin{array}{l}\text { Micro } \\
\text { Empresa }\end{array}$ & No Tiene \\
\hline 2012 & Mery & $\begin{array}{l}2 \text { Dos } \\
\text { Estrellas }\end{array}$ & 0 & 0 & 1 & $\begin{array}{l}\text { Micro } \\
\text { Empresa }\end{array}$ & No Tiene \\
\hline 2014 & Los Altos & $\begin{array}{l}3 \text { Tres } \\
\text { Estrellas }\end{array}$ & 7 & 9 & 1 & $\begin{array}{l}\text { Micro } \\
\text { Empresa }\end{array}$ & No Tiene \\
\hline 2014 & Hex Estelí & $\begin{array}{l}3 \text { Tres } \\
\text { Estrellas }\end{array}$ & 49 & 71 & 6 & $\begin{array}{l}\text { Pequeña } \\
\text { Empresa }\end{array}$ & www.hotelhex.com.ni \\
\hline 2015 & Vínculos & $\begin{array}{l}3 \text { Tres } \\
\text { Estrellas }\end{array}$ & 7 & 12 & 1 & $\begin{array}{l}\text { Pequeña } \\
\text { Empresa }\end{array}$ & No Tiene \\
\hline 2015 & Camilo`s & $\begin{array}{l}2 \text { Dos } \\
\text { Estrellas }\end{array}$ & 7 & 11 & 1 & $\begin{array}{l}\text { Micro } \\
\text { Empresa }\end{array}$ & No Tiene \\
\hline
\end{tabular}

Fuente: Elaboración propia a partir de los datos proporcionados por INTUR

Según la Ley 645 de incentivo a la MIPYME, las empresas se clasifican en microempresas cuando poseen de 1 a 5 trabajadores, pequeña empresa de 6 a 30 trabajadores y de 31 a 100 como mediana empresa tomando en cuenta el número de trabajadores. Por lo que el sector turístico hotelero está compuesto mayoritariamente: el $52.6 \%$ son microempresas y el $47.4 \%$ con pequeñas empresas.

Al analizar la conectividad a internet por cada categorización por Estrellas, tenemos que del total de los hoteles $43 \%$ no cuentan con conexión a internet, de los cuales $5 \%$ corresponde a categoría D, 21\% a 1 Estrella, $16 \%$ a 2 Estrellas y el $21 \%$ a 3 Estrellas, y solamente el 58\% de los hoteles cuentan con conexión a internet.

Según la encuesta el promedio mensual de visitantes tenemos que, el 26\% de los hoteles dicen tener un promedio de 26 a 50 visitantes mensual, el $21 \%$ expresa tener 76 a 100 y de 1 a 25 respectivamente y solamente el $16 \%$ menciona contar con más de 100 visitantes, cifras que corresponden con dato proporcionado por (Ramírez, 2016 ) quién expresó que al mes se tiene un promedio de turistas debidamente registrado y este va de 50 a 100.
Relacionándolo con la demanda del sector, según los datos de la encuesta de los turistas tenemos que solo el $40 \%$ de estos se hospedan en hoteles, baja afluencia promedio mensual de visitantes, ya que solo el $16 \%$ de los encuestados dicen tener más de 100 visitantes en promedio mensual, cabe mencionar que los propietarios expusieron que las afluencia de visitas varían cada mes, y lo consideran un servicio estacional.

Con respecto a la infraestructura en cantidad de habitaciones, el $42 \%$ de los hoteles tienen habitaciones en el rango de 1 a 10 habitaciones, el $21 \%$ de 11 a 20 , $11 \%$ de 21 a $30,16 \%$ de 31 a 40 , y solamente el $11 \%$ poseen más de 40 habitaciones.

Así mismo, los visitantes que reciben los hoteles son provenientes mayoritariamente turistas nacionales con un $58 \%$, de origen centroamericano con un $47 \%$, turistas norteamericanos con un $42 \%$ y minoritariamente Europeos. Esto lo podemos relacionar con el análisis de la demanda de alojamientos por parte de los turistas, en un $60 \%$ escogen otro tipo de alojamiento, tales como: hostales, casa huésped, hostal familiar, albergue, otros. Según, los datos obtenidos el tipo de turismo que reciben el sector hotelero es un turismo interno. 
Los tipos de visitantes según su estadía son visitantes que pernoctan de 1 a 3 días en un $73.7 \%$, de 4 a 6 días $5.3 \%$ y más de 6 días un $21.1 \%$.

El uso de las tecnologías de la información y comunicación se ha convertido en un elemento importante en la gestión de los negocios y en la manera de posesionarse en el mercado; buscar nuevos nichos de mercado, la facilidad de hacer negocios tanto con clientes como con proveedores, así mismo la creación de alianzas estratégicas para el negocio.

Para Sheldon (Citado en Figueroa \& Talon, 2006), el turismo es uno de los sectores con mayor orientación hacia la información y por ello no ha seguido ajeno al fenómeno de la adopción de innovaciones relacionadas a las TICs, al estar fuertemente sometido a la influencia de las mismas, llegando a convertirse en una industria intensiva en tecnología.

E1 95\% de los hoteles cuentan con una computadora para el negocio, este es un elemento muy importante, ya que la disposición de una computadora se convierte en un elemento importante para la aplicación de otras tecnologías en el negocio; como el acceso a internet o la automatización de diversos procesos internos.

Los medios de comunicación con los que cuenta el sector hotelero, en un 95\% red telefónica, $89 \%$ conexión a internet, el 42\% tecnología wap, 52\% telefonía móvil, y solo un $21 \%$ posee fax. El acceso a internet es un elemento importante en el proceso de negocio, ya que por medio de esta se tiene acceso a herramientas de marketing digital que se puede encontrar en la red. Otro aspecto importante es el uso de la telefonía móvil, ya que permite la integración de servicios audiovisuales, el acceso a internet desde el móvil, la realización de transacciones, y otros servicios que pueden ayudar y facilitar nuevas formas de utilización válidas para el ámbito empresarial.

\section{Sistemas de información en las funciones empresariales}

El acceso a la tecnología determina hoy en día la calidad de vida de la población y permite la competitividad del sector empresarial. De allí su importancia para el desarrollo de los hoteles en la ciudad de Estelí.

La gestión de un hotel requiere de muchas innovaciones y mejoras que permitan al establecimiento evolucionar y ofrecer una mejor calidad del servicio a los clientes, en la actualidad el mercado requiere que los hoteles adopten sistemas operativos compatibles con la tecnología móvil.

Sin embargo, en el estudio se determinaron barreras que dentro del sector hotelero implican un menor avance del negocio electrónico y que están fundamentalmente relacionadas con los siguientes aspectos: Problemas de seguridad (11\%); y los clientes u otras empresas no están preparados para la venta por internet (16\%).

Un poco más del $80 \%$ de los hoteles no consideran que existan barreras para hacer negocios por internet, así como el 100\% de los hoteles no creen que los servicios que prestan no son adecuados para la venta por internet. Aunque esta percepción está cambiando, precisamente por la propia evolución y demanda de los clientes en el sector, donde los hábitos de consumo de ocio han cambiado y las personas buscan una mayor comodidad y eficacia a la hora de reservar sus estancias.

Además, el estudio refleja la necesidad que los propietarios de los hoteles de implementar sistemas de gestión acordes con las necesidades del mercado actual.

Gran parte de los negocios que actualmente se realizan en el mundo se llevan a cabo a través de redes que conectan a las personas y a las compañías. Intranet es una red que conecta a las personas de una compañía entre sí y con la red de la empresa. Extranet conecta a una compañía con sus proveedores, distribuidores y otros socios externos. (Philip \& Armstrong, 2008) 
Cabe destacar, que la disponibilidad en el sector hotelero es del intranet $42 \%$ y extranet es del $37 \%$, lo que se traduce en la poca utilización de la red para distribuir información entre sus trabajadores y con proveedores y clientes. Entre los posibles usos del intranet está la posibilidad de desarrollar formación on line a sus empleados.

Los sistemas informáticos están compuestos por una matriz de componentes que colaboran entre sí, de manera que permita procesar información y producirla de manera organizada. Los sistemas informáticos cumplen diversos propósitos en una organización facilitando la gestión del negocio. (Effy Oz , 2008).

El sector hotelero en su mayor porcentaje (57.89\%) cuenta con algunos sistemas informáticos que le apoyan a la gestión del negocio. Sin embargo, un buen porcentaje de los hoteles del sector no disponen de ningún sistema informático (42.11\%).

Estos sistemas informáticos, corresponden a sistemas que facilitan en el proceso en el área de contabilidad (58\%), finanzas (26\%), mercadotecnia (16\%), Recursos Humanos (21\%), u otros (5\%):

Los sistemas informáticos en el área de contabilidad permiten registrar información y procesarla para producir informes financieros, registros de transacciones; estos informes permiten la toma de decisiones, de manera que se pueda cumplir los objetivos planteados. Así mismo, los informes financieros permiten medir y evaluar la rentabilidad de la aplicación de las estrategias de marketing digital; además de los indicadores de mercado como la relación precio/ganancia, entre otros. Así mismo, se puede realizar una previsión de inversión en Marketing digital.

Los sistemas informáticos en el área de mercadotecnia permiten analizar la demanda e identificar los grupos metas, de manera que se pueda precisar las estrategias de marketing digital que se aplicarán, así como su efectividad. De la misma manera, permite hacer proyecciones de demanda de los servicios e identificar como afecta la demanda la inversión en publicidad. Además, el sector hotelero puede analizar cómo se ha comportado la demanda del servicio a través del comportamiento de las reservaciones en diferentes periodos de tiempo, y puede vincularse a otras variables como el precio, la publicidad, etc.

\section{Uso de las TICS}

El internet es una inmensa red pública de redes de computadoras, este conecta a los usuarios de diversos tipos sin límite geográfico, esta red almacena una gran diversidad de información, la cual si se gestiona de manera adecuada es una herramienta muy útil para las empresas; ya que por medio del internet las empresas pueden expandir sus mercados.

En el sector hotelero el uso de internet es una herramienta indispensable, ya que los turistas tanto nacionales como extranjeros hacen uso del internet para la búsqueda de alojamiento, y es por medio del internet que se pueden hacer comparaciones de los distintos servicios que ofrece un hotel y buscar aquel que mejor se adapte a las necesidades de los clientes.

El uso del internet impulsa la creación de ventajas competitivas aquellos hoteles que por medio de la red hace uso de la información para gestionar sus procesos de negocios; tales como análisis del comportamiento de la demanda, principales tendencias del mercado, comportamiento de los precios del mercado. Así mismo, por medio de la conectividad del internet se puede crear estrategias digitales que les permita expandir sus mercados.

Uno de los principales usos del internet por parte del sector hotelero es la búsqueda de información para el negocio $(68 \%)$, realizar operaciones bancarias $(47 \%)$, venta del servicio (37\%), y por último, está la compra de enseres para el negocio (16\%). El uso del internet 
como herramienta para hacer negocios es importante, ya que el turista utiliza el internet en la búsqueda de alojamiento turístico y para realizar diversas transacciones desde la red, por ejemplo; reservas, contratación de paquetes turísticos, información del entorno y servicios de hoteles, etc.

El Internet permite configurar productos turísticos adaptados a diferentes colectivos y segmentos prioritarios en Internet: jóvenes, jubilados, solteros, etc.

En el aspecto de la publicidad el sector hotelero en mayor porcentaje utiliza el internet para realizar su publicidad (58\%), esto es una estrategia adecuada, ya que, analizado la demanda del sector, los turistas en un 50\% utilizan buscadores genéricos en internet para organizar su viaje.

\section{Aplicación de las Tecnologías de la información y la comunicación y su contribución a la competitividad.}

En este acápite se analiza las TICs que se aplican en las empresas del sector hotelero de Estelí y como estas contribuyen a la competitividad del sector.

\section{Aplicación de las TICs en los procesos empresariales} TICs en el Marketing y la comunicación

La competitividad de las empresas del sector, su capacidad de atraerclientes nacionales o internacionales, la eficiencia en la gestión comercial, está muy ligada al contar con nueva estrategia de Marketing on line.

El plan de marketing digital debe complementar el plan de marketing de la empresa, sin embargo, el mayor porcentaje de hoteles (53\%) no cuentan con un plan de Marketing bien definido, como se muestra en el gráfico siguiente:

El Marketing sirve para que las empresas puedan satisfacer las necesidades de los consumidores, las cuales se identifican a través de investigaciones de mercado, esto permite a las empresas establecer estrategias que permitirán conseguir los objetivos deseados. A continuación, en la siguiente tabla de contingencia podemos determinar que hoteles cuentas con un plan de Marketing bien definido. Por otro lado, el $68 \%$ de los hoteles dicen tener muy definido su segmento de mercado.

El segmento de mercado al que se dirige el sector hotelero son turistas nacionales en un $58 \%$ y el restante $42 \%$ son turistas extranjeros provenientes mayoritariamente de Norteamérica y Europa, como se muestra en la gráfica 28 , donde se puede observar el origen de los visitantes. La importancia de tener bien definido el segmento de mercado radica en la orientación al mercado de la estrategia de publicidad on line.

Las páginas Web de las cadenas hoteleras permiten obtener, la información sobre tarifas, descuentos en temporadas y niveles de ocupación, una visión completa de las instalaciones y servicios de los establecimientos que les son ofrecidos a los turistas para su alojamiento y servicios hoteleros complementarios.

La distribución del servicio está vinculado a los canales que se utiliza para llegar a los clientes, en este sentido el internet juega un rol importante en la venta del servicio on line. El desarrollo de un buen plan de marketing on line comienza con la creación de un sitio Web enfocado a distribuir y promocionar los servicios hotel. En este sentido se puede ilustrar en la gráfica 29 , que el $68 \%$ de los hoteles cuentan con página Web oficial del negocio y el $32 \%$ no cuenta con una página Web, es un porcentaje menor, sin embargo, es un porcentaje alto para el sector; ya que según la encuesta a los turistas el $58 \%$ de ellos utilizan el internet en especial buscadores genéricos como google para organizar su viaje.

Disponer una página Web brinda la oportunidad de automatizar la actividad del servicio al cliente, por lo que este servicio on line permite crear beneficios 
a los hoteles; tales como: la reducción de costos, ya que no se requiere de mucho personal para realizar esta actividad, así mismo lo clientes puede acceder a la Web las 24 horas del día, los 7 días de la semana. Por consiguiente, al reducir los costos los hoteles están creando ventajas competitivas.

En la página Web de los hoteles en su mayor porcentaje solo puede encontrar información general del hotel (68.42\%), en un mínimo porcentaje la página web tiene acceso a catálogos de servicios (21.05\%) y aplicaciones para el negocio, como plataformas para reservas (10.53\%). Estos datos son importante, ya que la página web es una herramienta útil para los hoteles, a través de esta se puede ofertar la variedad de los servicios, lista de precios y la posibilidad de tener una plataforma de reservas.

El marketing digital simplifica los procesos de marketing entre la empresa y los clientes, ahorra tiempo y agiliza las respuestas a las necesidades de los clientes. El uso intensivo de las TIC en el marketing hace que la empresa sea más innovadora, ya que percibe que su uso ayuda a superar las barreras a la innovación y acelera los procesos que, a su vez, ganan en eficiencia. (Grupo IFEDES S.A., 2007).

Las herramientas de Marketing Digital que más utilizan el sector hotelero es en mayor porcentaje (84.21\%) es en redes sociales, en específico el Facebook, seguido del E-mail Marketing (57.89\%), y en menor porcentaje la publicidad en blogs, google, SMS, entre otros. El sector hotelero tiene que valorar y retomar otras estrategias como el posicionamiento en buscadores como google, ya que el $50 \%$ de los turistas encuestados expresan que hacen uso de este tipo de herramienta para la organización de su viaje.

En la tabla siguiente se puede observar los elementos del marketing que aplican cada hotel del sector:

\begin{tabular}{|c|c|c|c|c|}
\hline HOTEL & $\begin{array}{l}\text { ¿El hotel cuenta con un plan } \\
\text { de marketing bien definido? }\end{array}$ & $\begin{array}{l}\text { ¿El hotel tiene bien definido } \\
\text { su segmento de mercado? }\end{array}$ & Internet & $\begin{array}{l}\text { ¿El hotel cuenta con una } \\
\text { página Web Oficial? }\end{array}$ \\
\hline Alameda & SI & SI & NO & SI \\
\hline Camilos's & SI & SI & SI & SI \\
\hline Campestre & NO & $\mathrm{NO}$ & NO & NO \\
\hline Cualitlan & NO & SI & SI & SI \\
\hline Don Vito & SI & SI & NO & SI \\
\hline El Chico & NO & SI & NO & NO \\
\hline El Mesón & SI & SI & SI & SI \\
\hline Estelimar & SI & SI & SI & SI \\
\hline Hex Estelí & SI & SI & SI & SI \\
\hline La Campiña & NO & SI & SI & si \\
\hline Las Américas & NO & $\mathrm{NO}$ & $\mathrm{NO}$ & SI \\
\hline Las Cornisas & NO & SI & SI & NO \\
\hline Los Altos & SI & SI & $\mathrm{NO}$ & SI \\
\hline Los Arcos & SI & SI & SI & SI \\
\hline Mery & NO & NO & SI & NO \\
\hline Panorama No. 1 & NO & $\mathrm{NO}$ & $\mathrm{NO}$ & NO \\
\hline Panorama No. 2 & NO & $\mathrm{NO}$ & $\mathrm{NO}$ & NO \\
\hline Puro Estelí & SI & SI & SI & SI \\
\hline Vínculos & NO & $\mathrm{NO}$ & SI & SI \\
\hline
\end{tabular}


De los datos de la tabla anterior, podemos analizar los porcentaje de los elementos del Marketing digital que aplican cada hotel, como se muestra en el siguiente gráfico: Los hoteles que cuentan con el $100 \%$ de los elementos necesarios para llevar a cabo las estrategias de Marketing digital son: El Mesón, Estelimar, Hex Estelí, Los Arcos, Puro Estelí y Camilos y aquellos hoteles que no poseen ningún elemento necesario para llevar a cabo las estrategias del Marketing Digital son el panorama No. 1 y No. 2; y Campestre.

\section{Gráfico $\mathrm{N}^{\circ}$ 1. Elementos del Marketing digital que aplican los hoteles}

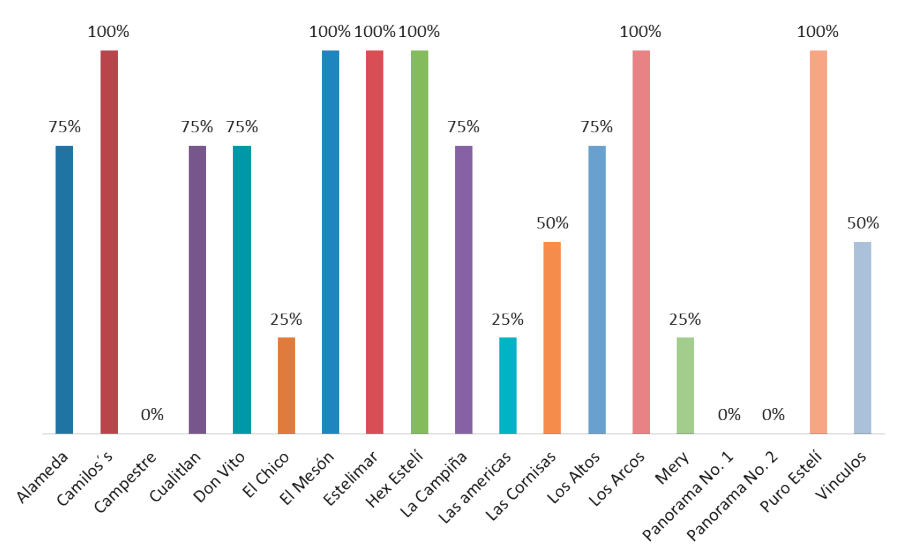

TICs y ventajas competitivas

Las razones por las que la información se puede convertir en una ventaja competitiva, son en primer lugar que a información es costosa de producir, pero barata de reproducir y de distribuir, y, en segundo lugar, que la información no desaparece cuando es consumida. (Figueroa \& Talon, 2006).

Dado el entorno en el que operan las empresas, el conocimiento se convierte en una fuente de ventaja competitiva.

Las razones por lo que el sector hace uso del interne para la venta es por: captación de nuevos clientes (63\%), mejora la imagen del hotel y mantenerse a la altura de los competidores (42\%), expansión geográfica del mercado (26\%), y para acelerar el proceso de negocios $(16 \%$.)

Otro elemento importante para la creación de ventajas competitivas es poder captar clientes y expandir tu mercado es a través de poder utilizar herramientas útiles que reduzcan los costos. En este sentido los hoteles del sector valoran cada tipología de Marketing digital por su grado de eficiencia en términos de costos; la publicidad en redes sociales (37\% como eficiente), E-Mail Marketing (32\% como eficiente), y un 5\% dice que la publicidad en redes sociales es ineficiente.

Del gasto total en Marketing, los hoteles del sector destinan en inversión un porcentaje muy pequeño de 1 a 25\% (89\%), solo el 5\% de los hoteles invierten en el rango del 26 al 50\%.

Lo anterior se puede relacionar con aspectos como; contar con plataformas on line para reservas, ya que solo el $53 \%$ poseen y $47 \%$ no cuentan con una plataforma on line que les permita realzar sus reservas. La importancia de utilizar plataformas intermediarias de reservas radica en que este tipo de plataforma (Booking, Trivago, TripAdvisor, etc) crea foros y blogs de turismo, ya que los clientes comentan y dan puntaje a los hoteles generando un modelo de promoción social.

Las plataformas web que más utilizan para intermediar su servicio son Booking (47.37), TriAdvisor (42.11\%); en menor porcentaje trivago, los viajes.com y otras plataformas como Vianica.

Los hoteles que hacen uso o no de alguna plataforma on line de reservas o alguna plataforma intermediaria, aquí es importante mencionar que los hoteles que no hace uso de ninguna plataforma on line de reserva son el Campestre, Cualitlan, Las Americas, Panorama No1 y No 2. Estos datos son importantes porque el uso de plataformas on line de reservas que aportan a la competitividad, ya que constituye un contacto 
directo con los clientes, evitando la necesidad de los intermediarios que encarecen el proceso de ventas.

Para la obtención de la ventaja competitiva los hoteles pueden crear alianzas estratégicas con otros prestadores de servicios de manera que les permita combinar sus servicios de manera que les permita hacerlos más atractivos y menos costosos. Internet permite el "asociarse virtualmente" con otras compañías del sector como transporte (autobuses, taxis, etc.), ocio y cultura (deportes de aventura, cine y teatros, etc.), u otros servicios de establecimientos hoteleros. Este asociacionismo genera un valor sobre el producto por facilitar una oferta más rica e integrada de servicios turísticos.

Con respecto a las alianzas estratégicas que poseen los hoteles, solamente el $36.38 \%$ dijo tener algún tipo de alianzas; entre las que se destacan están las alianzas con los proveedores $(26.32 \%)$, toor operadoras turísticas (26.332\%), Agencias publicitarias (15.79\%), Agencias de viaje (15.79\%), en menor porcentaje con aerolíneas, entidades financieras y otros.

Cabe señalar que en la actualidad las empresas del sector turístico hotelero hacen un gran esfuerzo por sobrevivir dentro del mercado; sin embargo, a la mayoría se les dificulta invertir en implementar estrategias de crecimiento costosas, entre las cuales se encuentran las que tienen que ver con el área de mercadotecnia. Por lo general, las empresas cuando quieren aplicar cualquier plan de acción relacionado con la promoción y publicidad de productos o mejorar su posicionamiento, recurren a empresas externas especializadas (agencias de publicidad) que por lo general son costosas y no todas tienen la posibilidad de hacer esa inversión. El hecho de no implementar estrategias de mercadotecnia bien presupuestadas, lejos de beneficiar, puede ser perjudicial para una pequeña empresa turística hotelera porque es una inversión no planificada.
Además, las TIC son esenciales para mejorar la productividad de las empresas, la calidad, el control y facilitar la comunicación entre otros beneficios, aunque su aplicación debe llevarse a cabo de forma inteligente. (EURECA, 2012).

En este sentido el sector turístico hotelero debe tomar la decisión de introducir tecnología digital y en la cual se garantice y aproveche al máximo esta como una ventaja competitiva, pero si se puede considerar como la puerta a la inserción a nuevos mercados. Para que la empresa logre la implantación de nueva tecnología y esta produzca efectos positivos hay que cumplir varios requisitos (EURECA, 2012):

- Tener un conocimiento profundo de los procesos de la empresa

- Planificar detalladamente las necesidades de tecnología de la información

- Incorporar los sistemas tecnológicos paulatinamente, empezando por los más básicos.

Como anteriormente se explica la implementación de tecnología en los procesos trae consigo una serie de consideraciones, pero igualmente trae ventajas en el marketing digital que se pueden mencionar como las siguientes:

- Apoyar a los empresarios locales para presentar y vender sus productos a través de Internet.

- Permitir el aprendizaje interactivo y la educación a distancia para los empleados

- Impartir nuevos conocimientos para la empleabilidad que requieren muchas competencias (integración, trabajo en equipo, motivación, disciplina, etc.).

- Ofrecer nuevas formas de trabajo y de inclusión laboral, como teletrabajo

- Dar acceso al flujo de conocimientos e información para empoderar y mejorar las vidas de las personas, facilidades, exactitud, menores riesgos, menores costos, etc.

- Mejora de procedimientos internos de trabajo.

- Incremento y mejora de las relaciones con clientes 
y proveedores.

- Creación de nuevos canales de distribución.

- Mayor flexibilidad y agilidad de las empresas frente al cambio

\section{Propuesta de estrategias de Marketing Digital para los hoteles de la cuidad de Estelí.}

Estrategia 1: Fidelización de clientes

Objetivo: Mantener a los clientes actuales, así como aumentar a clientes atraídos por los descuentos por el consumo.

\section{Actividades:}

1. Creación de tarjeras que permitan a los clientes acumular puntos para obtener regalos o descuentos en futuras compras.

2. Cupones de descuento tanto para el pago de contado como para las compras por internet.

3. Aplicación de un sistema informático que permita llevar un control de los puntos acumulados $\mathrm{y}$ cupones de descuentos.

4. Crear un base de datos de los clientes que permita profundizar en el conocimiento sobre ellos para individualizar.

5. reación de club de clientes prioritarios, estos tendrán acceso a una tarjeta de fidelización que permita identificar a los miembros del club.

6. Compartir información con los clientes por medio de sitios web, e.mail, etc.

7. Tomando en cuenta la información que se posee de los clientes (edad, sexo, nivel educativo, gustos, etc.) se puede enviar comunicación adaptadas a sus necesidades consiguiendo una personalización.

Estrategia 2: Posicionamiento Web.

Objetivo: Conseguir que el sitio web este priorizado por los buscadores y obtenga mayor popularidad y relevancia.

\section{Actividades:}

1. Vincular el sitio web con sitios web de referencia acordes a la temática de nuestro sitios Web, es decir con buscadores on line de reservas de alojamientos; tales como trivago, booking, vianica, etc.

2. Hermanarse o intercambiar enlaces con otras Web de alojamientos.

3. Incluir los sitios Web en sitios directorios clasificados.

4. Crear perfiles en redes sociales como facebook y twiter.

Estrategia 3: Monitoreo de la marca por medio de las redes sociales

Objetivo: Conocer la imagen hacia el exterior de manera que permita tomar las decisiones oportunas.

Conocer los gustos y preferencias de los clientes buscando en las respuestas que expresan respecto al negocio.

\section{Actividades:}

1. Monitorizar las redes sociales a través de herramientas que permiten monitorizar los resultados en las redes sociales, en las cuales se puede monitorizar en toda la red sobre la marca, medir el sentimiento de estas conversaciones, integración de analítica Web a través de Google Analytics, Webtrends y Omniture; así como software de gestión al servicio al cliente como Salesforce. Existen herramientas gratuitas como ThinKup o de pago como Radian 6, Alterian SM2, SocialBro, etc.

2. Gestionar la reputación on line de la marca y conseguir nuevos clientes: Hacer estudios sobre lo que sucede alrededor de la marca y su imagen hacia el exterior. Existen herramientas gratuitas tales como; Socialmention, Howsociable, Postrank, etc.; y de pago como; Shotools.

3. Gestión de perfiles a través de herramienta que permita integrar las redes sociales. Existen 
herramientas gratuitas, tales como; TweetDeeck, Sobees, Seesmic; y de pago como; Cotweet, Postling, etc.

Estrategia 4. Gestión de precios

Objetivo: Obtener una relación equitativa entre las necesidades de los clientes y el beneficio del negocio.

\section{Actividades:}

1. Llevar un diario de precios, en el cual se registre cronológicamente una serie de aspectos que afectan los precios, tales como; estacionalidad, eventos, número de reservas producidas al día, variación de las reservas, cantidad de noches vendidas con respectos a las reservas producidas. Existen herramientas, como Ruralgest que es un software que permite conocer la disponibilidad, los días mínimos de reservas, etc.

2. Elaborar pronóstico de reservas mensual, de manera que permita analizar la demanda en determinado periodo de tiempo.

3. Elaborar curvas de demanda según segmentos de clientes (para lo cual se deben hacer segmentos de clientes, con parámetros como el sexo, nacionalidad, etc.).

Estrategia 5: Estudios de competencia

Objetivo: Observar el comportamiento de la competencia para determinar si sus políticas de actuación han sido correctas, si les ha llevado a un mayor volumen de ingresos y si los clientes están satisfechos con el producto que ofertan.

\section{Actividades:}

1. Monitorear por internet la información relativa a los posibles competidores del sector. Mediante blogs, prensa especializada, reportes del sector donde se pueden observar los principales indicadores de éxito y fracaso de la competencia mediante el uso de foros hoteleros se puede dar seguimiento de las opiniones y comentarios de los clientes.
Estrategia 6: Gestión de clientes y envío de boletines

Objetivo: Llevar un control de los clientes y tener organizada la información de los clientes.

\section{Actividades:}

1. Crear una base de datos de los clientes.

2. Agrupar a los clientes según sus necesidades de alojamiento.

3. Enviar a los clientes, mediante boletines, las mejores ofertas.

\section{CONCLUSIONES}

Al analizar la opinión de los turistas, los propietarios y/o gerentes de los hoteles, se llegó a las siguientes conclusiones:

En cuanto a la caracterización del sector:

- La demanda del sector turístico hotelero está compuesto por visitantes relativamente jóvenes, con un nivel de educación alto y su principal motivo de la visita es por vacaciones, así como su tiempo de estadía va de dos a cinco días.

- En cuanto a los medios de información sobre la ciudad, los turistas utilizan internet, en su mayoría escogen otro tipo de alojamiento distinto al hotel esto obedece más que todo a la variable precio. Para la búsqueda del alojamiento turístico utilizan el internet y organizan su viaje a través de organización propia.

- Por otra parte, la oferta de alojamientos turístico hotelero el sector está compuesto por microempresas con categorías de 1 a 3 Estrellas con una capacidad hotelera de 1 a 10 habitaciones.

- El origen de los visitantes es mayoritariamente Nacional, por lo que el tipo de turismo es interno. En cuanto a la conectividad del internet el mayor porcentaje de los hoteles cuentan con conexión. 
Con relación a las tecnologías aplicadas en sector turístico hotelero:

- El mayor porcentaje de los hoteles disponen de computadoras personal disponibles para la gestión del negocio, cuentan con los medios de comunicación tales como: red telefónica, conexión a internet, telefonía móvil, etc. Las barreras que el sector identifica para hacer negocios por internet es mayoritariamente que existen problemas de seguridad y que los clientes u otras empresas no están preparados para la venta por internet. En su mayoría no cuentan con software internos y externos que le ayude a la gestión del negocio.

- Por otra parte, el mayor porcentaje de los hoteles no cuentan con un sistema informático para la gestión del negocio, en su mayoría poseen solo un sistema de para la contabilidad.

- El uso que le dan al internet es mayoritariamente para búsqueda de información relevante para el negocio. Los medios publicitarios que utilizan es por medio de internet.

En cuanto a la aplicación de las TIC y su contribución a la competitividad del sector:

- En su mayoría las empresas del sector no cuentan con un plan de marketing bien definido. Sin embargo, si tienen claro el segmento de mercado al que se dirigen.

- En su mayoría los hoteles cuentan con una página Web corporativa, en la cual se puede observar alguna información general del negocio; tales como la presentación, algunas aplicaciones para el negocio como gestión de reservas y el acceso a catálogos de servicios.

- Los hoteles apuestan por la publicidad por medio de las redes sociales y hacen uso del e-mail Marketing, consideran que estos medios son más eficientes en términos de costos.

- Las razones por las que hacen uso de la venta por internet es para captar nuevos clientes, mantenerse a la altura de los competidores y mejorar la imagen del hotel.
- Los hoteles en su mayoría no destinan mucha inversión en Marketing digital, por lo que hacen uso de las redes o web disponibles de manera gratuita.

- En su mayoría cuentan con una plataforma on line de reservas, sin embargo, existen un gran porcentaje que aún no lo hace; así mismo utilizan algunas plataformas web intermediarias de reservas tales como; TripAdvisor, Booking, etc.

- Así mismo, los hoteles cuentan con algunas alianzas estratégicas entre las que se destacan, los proveedores y tour Operadoras.

En cuanto a las estrategias de Marketing digital que contribuyan a la competitividad:

A nivel del ambiente interno y externo se presentan oportunidades que el sector puede aprovechar, sobre todo dado los avances tecnológicos. Existen plataformas o herramientas on line tanto gratuitas como pagadas que los hoteles pueden aprovechar y que le ayude a gestionar el Marketing del negocio a través de la web.

En cuanto al cumplimiento de la hipótesis, podemos decir, que el uso y aplicación de las TICs contribuye a que el sector hotelero incremente su competitividad a través de la expansión a nuevos mercados, la reducción de costos haciendo uso de plataformas on line; además permite tener más contacto directo con los clientes de manera eficiente, entre otros. Sin embargo, todavía en el sector turístico hotelero existen un considerable porcentaje que no hace uso de estas herramientas de Marketing Digital.

\section{RECOMENDACIONES}

Los objetivos del presente estudio es poder ofrecer al sector turístico hotelero recomendaciones que permitan a los propietarios y gerentes abordar con mayor confianza los proyectos de introducción de tecnologías de la información y la comunicación (TIC) y la 
explotación correcta de su sistema de información. Por lo tanto, en este acápite se brindan recomendaciones a partir de las conclusiones mencionadas anteriormente:

- El sector hotelero debe de introducir nuevas tecnologías que presenten ventajas competitivas de forma correcta.

- Deben de gestionar recursos para realizar la inversión y además acompañar este proceso de su adopción con cambios en su organización y en el funcionamiento de la empresa.

- Se debe de formar a los empleados como usar los diferentes sistemas para promocionar sus servicios y brindar correcta atención a sus clientes.

- Debido a que en el sector turístico los productos y servicios que se ofrecen generan información y conocimiento desde el primer contacto con los clientes, es importante que los gerentes o propietarios identifiquen la importancia de manejar $\mathrm{y}$ difundir información relevante $\mathrm{y}$ oportuna mediante las TIC.

- Las Tecnologías de Información pueden ayudar a mejorar la productividad de todas las funciones de la empresa y el flujo de información dentro y entre las unidades de cada hotel.

- Es recomendable que los sistemas Web turísticos de los hoteles incorporen otras estrategias que les signifiquen un valor agregado y representen una ventaja competitiva frente a otros sectores turísticos hoteleros de otras regiones y países.

- INTUR debe de incluir en su plan acciones que contribuyan a la publicidad y promoción del marketing digital del sector, tales como: asesoramiento a la industria turística hotelera en materia de TIC, desarrollo de estándares nacionales de competencia sobre TIC, dar los lineamientos respecto a la infraestructura básica necesaria para sustentar adecuadamente la implantación de TIC, entre otros.

- Se deben diseñar páginas en la web, que sean accesible y de bajo costos para el sector turístico hotelero.

- Establecer alianzas con universidades e INTUR para el diseño de sus páginas en la web.

- Por último, el sector turístico hotelero debe de aprovechar de forma correcta las diferentes plataformas o herramientas existentes on line, tanto gratuitas como pagadas, de tal forma que les ayude a gestionar el Marketing del negocio a través de la web.

\section{BIBLIOGRAFÍA}

Effy Oz . (2008). Administración de los sistemas de información. México, D.F: Cengage Learning Editores, S.A.

EURECA. (2012). Gestión de las Tic en las MIPYMES. Estelí: Isnaya.

Grupo IFEDES S.A. (2007). Las TIC en la estrategia empresarial. Valencia: Anetcom.

Hernández Sampierí, R., Fernández Collado, C., \& Baptista, P. (2010). Metodología de la investigación. (Quinta edición ed.). México: McGraw Hill.

Kotler, P. (2008). Fundamentos de Marketing. México: Person Educación.

López, J. P. (2008). Metodología de la investigación científica (Sexta ed.). Managua, Nicaragua.

Mendez A., C. (2009). Metodología, diseño y desarrollo del proceso de investigación con enfasis en las ciencias empresariales. México: LIMUSA.

Philip, K., \& Armstrong, G. (2008). Fundamentos de Marketing. México : Pearson Educatión .

Ramírez, L. (2016 , Mayo 02). Delegada de INTUR . (S. Alonso, Interviewer)

Tamayo, R. (1994). El proceso de investigación cientifica. México: LIMUSA-Noriega editores.

Yuni, J. A., \& Urbano, C. (2006). Técnicas para investigar: recursos metodológicos para la preparación de proyectos de investigación. Argentica: Brujas. 